\title{
Serine/threonine protein kinases PknF and PknG of Mycobacterium tuberculosis: characterization and localization
}

\author{
Anil Koul, ${ }^{1,3,5}$ Axel Choidas, ${ }^{2}$ Anil K. Tyagi, ${ }^{3}$ Karl Drlica, ${ }^{4}$ Yogendra Singh ${ }^{1}$ \\ and Axel Ullrich ${ }^{5}$
}

Author for correspondence: Yogendra Singh. Tel: +91 11766 6156. Fax: +91 117667471. e-mail: ysingh@cbt.res.in

1 Centre for Biochemical Technology, Mall Road, Delhi-110 007, India

2 Axxima Pharmaceuticals AG, Am Klopferspitz 19, 82152 Martinsried, Germany

3 Department of Biochemistry, University of Delhi South Campus, N. Delhi, India

4 Public Health Research Institute, 455 First Avenue, NY, USA

5 Department of Molecular Biology, Max-PlanckInstitut für Biochemie, Am Klopferspitz 18A, 82152 Martinsried, Germany

\begin{abstract}
Pathogenesis of Mycobacterium tuberculosis is closely connected to its survival and replication within the host. Some pathogenic bacteria employ protein kinases that interfere with the cellular signalling network of host cells and promote bacterial survival. In this study, the pknF and pknG genes, which encode two putative protein kinases of $M$. tuberculosis $H_{37} R v$, protein kinase $F$ (PknF) and protein kinase G (PknG), respectively, were cloned and expressed in Escherichia coli. Purified PknF phosphorylated the peptide substrate myelin basic protein (MBP) at serine and threonine residues, while purified PknG phosphorylated only at serine residues. The activity of the two kinases was abrogated by mutation of the codon for the predicted ATP-binding-site lysine residue. Southern blot analysis revealed that homologues of the genes encoding the two kinases are present in $M$. tuberculosis $H_{37} R$ a and Mycobacterium bovis BCG, but not in Mycobacterium smegmatis. Immunoblot analysis of various cellular fractions of $M$. tuberculosis $H_{37} R v$ revealed that PknF is a transmembrane protein and that PknG is predominantly a cytosolic enzyme. The present study should aid in elucidating the role of these protein kinases in the pathogenesis of mycobacteria.
\end{abstract}

Keywords: tuberculosis, protein phosphorylation, glutathione $S$-transferase, transmembrane, mycobacteria

\section{INTRODUCTION}

Mycobacterium tuberculosis, the causative agent of tuberculosis, employs several strategies for survival in host cells. Among these are inhibition of phagosomelysosome fusion (Armstrong \& Hart, 1975), inhibition of acidification of phagosomes (Sturgill-Koszycki et al., 1994), resistance to killing by reactive oxygen and nitrogen intermediates (Lowrie, 1983), and recruitment and retention of tryptophan/aspartate-containing coat protein (TACO) on phagosomes to prevent their delivery to lysosomes (Ferrari et al., 1999). Bacterial and host factors signalling and controlling these events are poorly understood.

The regulation of eukaryotic signal transduction pathways by phosphorylation-dephosphorylation of serine, threonine and tyrosine residues has been known for many years (Yarden \& Ullrich, 1988). Recently, protein

Abbreviations: GST, glutathione S-transferase; MBP, myelin basic protein. kinases have also been found to coordinate stress responses, development processes and pathogenicity in several micro-organisms (Av-Gay \& Everett, 2000). For example, Ser/Thr kinase (YpKA) is involved in the expression of virulence in Yersinia pseudotuberculosis (Galyov et al., 1993) by disrupting the eukaryotic cytoskeleton and by reprogramming the host signalling network (Hakansson et al., 1996).

The presence of functional Ser/Thr kinases (Peirs et al., 1997) and phosphorylated protein (Chow et al., 1994) in mycobacteria was reported prior to release of the complete genome sequence of $M$. tuberculosis. The genomic sequence then suggested the presence of 11 putative protein kinases and 4 protein phosphatases (Cole et al., 1998). Although the role of these mycobacterial kinases is not yet clear, they may be regulators of various metabolic processes, growth, development and interaction with host cells. Recently, inhibitors of protein kinases have been shown to prevent uptake of Mycobacterium leprae by peritoneal macrophages of mice (Prabhakaran et al., 2000). 
Recently, two putative protein Tyr phosphatases (Koul et al., 2000) and two functional Ser/Thr kinases, PknD (Peirs et al., 1997) and PknB (Av-Gay et al., 1999), from M. tuberculosis have been cloned and characterized. The present work describes the cloning, expression and characterization of PknF and PknG, two more Ser/Thr protein kinases of M. tuberculosis. The data show that $p k n F$ and $p k n G$ code for functional kinases, that $P k n F$ is a transmembrane protein, and that PknG is a cytosolic enzyme.

\section{METHODS}

Bacterial strains and plasmids. Proteins from M. tuberculosis $\mathrm{H}_{37} \mathrm{Rv}$ cell wall, cell membrane and cytosolic subcellular fraction, and cell lysates of M. tuberculosis $\mathrm{H}_{37} \mathrm{Rv}$ and $\mathrm{H}_{37} \mathrm{Ra}$ were provided by John T. Belisle under the Tuberculosis Research Material and Vaccine Testing Program of the National Institute of Allergy and Infectious Diseases, National Institute of Health, CO, USA (contract no. AI-75320). Genomic DNA was isolated from M. tuberculosis $\mathrm{H}_{37} \mathrm{Rv}, M$. tuberculosis $\mathrm{H}_{37} \mathrm{Ra}$, Mycobacterium bovis BCG and Mycobacterium smegmatis $\mathrm{mc}^{2} 155$. M. tuberculosis $\mathrm{H}_{37} \mathrm{Rv}, M$. tuberculosis $\mathrm{H}_{37} \mathrm{Ra}$ and $M$. bovis BCG were grown in $7 \mathrm{H} 9$ medium containing $10 \%$ albumin dextrose complex (ADC) at $37^{\circ} \mathrm{C}$ for 3-4 weeks with shaking at 220 r.p.m. M. smegmatis was grown in Middlebrook $7 \mathrm{H} 9$ medium (Difco) supplemented with $0 \cdot 2 \%$ glucose at $37^{\circ} \mathrm{C}$ for $2 \mathrm{~d}$. The expression vectors pGEX-5X-3 and pCDNA3 were obtained from Pharmacia Biotech and Invitrogen, respectively. Enhanced Chemiluminescence (ECL) reagent was obtained from NEN Research Products.

Plasmid construction and mutagenesis. M. tuberculosis $\mathrm{H}_{37} \mathrm{Rv}$ genomic DNA was used as a template for amplification of two putative protein kinase genes, $p k n F$ and $p k n G$, by PCR. The sequences of the two PCR primers for cloning $p k n F$ were 5'-GGAATTCCATGCCGCTCGCGGAAGGTTCAACGTTCC-3' for the $5^{\prime}$ end (carrying an EcoRI site) and 5'-CCGCTCGAGTCACGGCCAGCCGTTGCTTCTGCG$3^{\prime}$ for the $3^{\prime}$ end (carrying an XhoI site). For cloning $p k n G$, the sequences of the two primers were $5^{\prime}$-GGAATTCCATGGCCAAAGCGTCAGAGACCGAAC-3' for the $5^{\prime}$ end (carrying an EcoRI site) and 5'-CCGCTCGAGTTAGAACGTGCTGGTGGGCCGGAC-3' for the $3^{\prime}$ end (carrying a $X h o I$ site). The amplified products of $p k n F$ and $p k n G$ were digested with EcoRI and XhoI, and the resulting fragments were inserted into pGEX-5X-3, which was previously digested with the same restriction enzymes. The resulting plasmids were designated pGEX- $p k n F$ and pGEX- $p k n G$.

Site-directed mutagenesis of lysine-41 of $\mathrm{PknF}$ and lysine-181 of PknG to methionine was carried out as described by Kunkel et al. (1991). The oligonucleotide used for mutagenesis of $p k n F$ was 5'-GGCCCGCAGTACCATGAGCGCGTCCTG$3^{\prime}$, and the oligonucleotide for mutagenesis of $p k n G$ was $5^{\prime}$ ATGCACCAGGCCCATGAGCACCACCGG-3' (underlined bases indicate the change from lysine to methionine). The plasmids carrying the mutant genes were designated pGEX$p k n F-\mathrm{K} 41 \mathrm{M}$ and pGEX-pknG-K181M. The $p k n F$ gene was also cloned into a eukaryotic expression vector, pCDNA3, and the resulting plasmid was designated pCDNA3- $p k n F$. The nucleotide sequences were confirmed by sequencing using the dideoxynucleotide method (Sanger et al., 1977).

Expression and purification of PknF and PknG. Escherichia coli strain BL21 was separately transformed with plasmids pGEX-pknF, pGEX-pknF-K41M, pGEX-pknG and pGEX-
pknG-K181M. Transformants were grown in 2YT medium (Difco) containing $100 \mu \mathrm{g}$ ampicillin $\mathrm{ml}^{-1}$ at $37^{\circ} \mathrm{C}$ until the $\mathrm{OD}_{600}$ reached $0 \cdot 5$. IPTG was then added to a final concentration of $0.5 \mathrm{mM}$, and cultures were grown for an additional $5 \mathrm{~h}$ at $37^{\circ} \mathrm{C}$ with shaking. Cells were harvested by centrifugation at $5000 \mathrm{~g}$ for $15 \mathrm{~min}$ and resuspended in $20 \mathrm{ml}$ sonication buffer $(50 \mathrm{mM}$ Tris/ $\mathrm{HCl} \mathrm{pH} 7 \cdot 4,150 \mathrm{mM} \mathrm{NaCl}$, $1 \mathrm{mM}$ EDTA, $10 \%$ (v/v) glycerol, $1 \mathrm{mM}$ PMSF and $10 \mu \mathrm{g}$ aprotinin $\mathrm{ml}^{-1}$ ). The cells were then sonicated on ice for $2 \mathrm{~min}$, and the sonicate was supplemented with Triton X-100 to a final concentration of $1 \%$ before centrifugation at $30000 \mathrm{~g}$ for $30 \mathrm{~min}$ at $4{ }^{\circ} \mathrm{C}$. The supernatant fluid was incubated overnight at $4{ }^{\circ} \mathrm{C}$ with glutathione-Sepharose $4 \mathrm{~B}$ matrix (Pharmacia Biotech). The resin bound to protein was packed into a column and washed with 5 bed vols PBS (1.44 g disodium hydrogen phosphate, $0.24 \mathrm{~g}$ potassium dihydrogen orthophosphate, $0 \cdot 2 \mathrm{~g}$ potassium chloride, $8.0 \mathrm{~g}$ sodium chloride in 1 litre distilled water, $\mathrm{pH} 7 \cdot 2$ ). Proteins were eluted with $50 \mathrm{mM}$ Tris $/ \mathrm{HCl} \mathrm{pH} 8.0$ containing $1 \mathrm{mM}$ DTT, $5 \mathrm{mM}$ $\mathrm{MgCl}_{2}$ and $15 \mathrm{mM}$ glutathione. Fractions were analysed by $10 \%$ SDS-PAGE (Laemmli, 1970). Fractions containing purified chimeric proteins were pooled and dialysed against PBS containing $20 \%$ glycerol and stored at $-20{ }^{\circ} \mathrm{C}$.

PknF and PknG kinase activities. The kinase assay is based on the phosphorylation of myelin basic protein (MBP). The activities of wild-type and mutated PknF and PknG were determined by incubating $0.5 \mu \mathrm{g}$ glutathione $S$-transferase (GST)-PknF, GST-PknF-K41M, GST-PknG or GST-PknG$\mathrm{K} 181 \mathrm{M}$ with MBP $(1 \mu \mathrm{g})$ for $30 \mathrm{~min}$ at $37^{\circ} \mathrm{C}$ in a kinase buffer (25 mM Tris/HCl pH 7.4 containing $5 \mathrm{mM} \mathrm{MgCl}_{2}, 2 \mathrm{mM}$ $\mathrm{MnCl}_{2}, 1 \mathrm{mM}$ DTT, $200 \mu \mathrm{M}$ orthovanadate and $3.7 \times 10^{5} \mathrm{~Bq}$ $\left.\left[\gamma^{32} \mathrm{P}\right] \mathrm{ATP}\right)$. The reactions were terminated by the addition of SDS sample buffer and proteins were separated by $15 \%$ SDSPAGE. The gels were electroblotted to nitrocellulose membranes and autoradiographed to determine the phosphorylation of MBP.

Analysis of phosphorylated residues of MBP. Analysis of phosphoamino residues of MBP phosphorylated by $\mathrm{PknF}$ or PknG was carried out as described by Vincent et al. (1999). In brief, MBP was phosphorylated by either PknF or PknG, and the protein components were separated by $15 \%$ SDS-PAGE. They were then electroblotted to a PVDF membrane. The bands corresponding to the phosphorylated MBP were excised from the membrane and acid-hydrolysed in $5.7 \mathrm{M} \mathrm{HCl}$ for $90 \mathrm{~min}$ at $110^{\circ} \mathrm{C}$. The acid-stable phosphoamino acids liberated by hydrolysis were separated by two-dimensional TLC and detected by autoradiography.

Production of polyclonal antiserum to PknF and PknG. Purified GST-PknF or GST-PknG chimeric proteins $(150 \mu \mathrm{g})$ were dissolved in $1 \mathrm{ml}$ Freund's incomplete adjuvant and injected into rabbits. Subsequently, injections of $100 \mu \mathrm{g}$ each of GST-PknF or GST-PknG in $1 \mathrm{ml}$ Freund's incomplete adjuvant were given three times at $14 \mathrm{~d}$ intervals. Ten days after the final injection, animals were bled, and titres of anti GST-PknF were determined by ELISA as described by Harlow \& Lane (1988).

Expression of PknF in A549 cell line. The human lung epithelial cell line A549 was maintained in Ham's F12 medium (GibcoBRL) supplemented with $10 \%$ fetal calf serum. Cells were transfected with pCDNA3 alone or the pCDNA3-pknF construct by the calcium phosphate method (Chen \& Okayama, 1987) and lysed as described previously (Zwick et al., 1999).

Southern blot analysis. Analysis of the presence of $p k n F$ and $p k n G$ homologues in various species of mycobacteria was 
carried out as described by Reyrat et al. (1995). In brief, genomic DNA samples (3 $\mu \mathrm{g}$ each) from $M$. tuberculosis $\mathrm{H}_{37} \mathrm{Rv}$, M. tuberculosis $\mathrm{H}_{37} \mathrm{Ra}, M$. bovis BCG and $M$. smegmatis were digested with Bam HI/NruI (for hybridization with $p k n F$ ) or with SmaI (for hybridization with $p k n G$ ). Digested products were separated by electrophoresis in a $1 \%$ agarose gel at $25-30 \mathrm{~V}$ for $16 \mathrm{~h}$ and transferred to nitrocellulose membranes. Hybridization was performed at $66^{\circ} \mathrm{C}$ using $6 \times \mathrm{SSC}(1 \times \mathrm{SSC}$ is $150 \mathrm{mM} \mathrm{NaCl}$ and $15 \mathrm{mM}$ sodium citrate, $\mathrm{pH} 7 \cdot 2$ ) with a ${ }^{32} \mathrm{P}$-labelled $p k n F$ probe or with a ${ }^{32} \mathrm{P}$ labelled $p k n G$ probe. After washing with $0 \cdot 1 \times$ SSC and $0 \cdot 1 \times \operatorname{SDS}$ for $20 \mathrm{~min}$ at $68^{\circ} \mathrm{C}$, hybrids were detected by autoradiography.

\section{RESULTS}

\section{Expression and purification of PknF and PknG}

Genome sequence data of $M$. tuberculosis revealed the presence of 11 putative protein kinases (Cole et al., 1998). Two genes with sequence homologies to protein kinases, $p k n F(\mathrm{Rv}$ 1746) and $p k n G$ (Rv 0410c) were amplified by PCR from genomic DNA of M. tuberculosis $\mathrm{H}_{37} \mathrm{Rv}$ using oligonucleotide primers deduced from the genome sequence of M. tuberculosis (Cole et al., 1998). The amplified products were cloned in pGEX-5X-3. The resulting plasmids, designated pGEX-pknF or pGEX$p k n G$, were used to transform E. coli.

The expressed GST-chimeric proteins (GST-PknF and GST-PknG) were purified using glutathione-Sepharose 4B matrix. The GST-PknF and GST-PknG chimeric proteins migrated in $10 \%$ SDS-PAGE as a $93 \mathrm{kDa}$ protein (Fig. 1a, lane 2) and a $111 \mathrm{kDa}$ protein (Fig. 1b, lane 2), respectively. The predicted size of GST-PknF was $80 \mathrm{kDa}(51 \mathrm{kDa}$ for the PknF protein and $29 \mathrm{kDa}$ for the attached GST protein). The size of the GST$\mathrm{PknG}$ chimeric protein was consistent with the predicted molecular mass of the PknG protein $(82 \mathrm{kDa})$ plus the attached GST protein $(29 \mathrm{kDa})$. The mutated chimeric proteins, GST-PknF-K41M (Fig. 1a, lane 3) or GSTPknG-K181M (Fig. 1b, lane 3), were purified by the same procedure.

\section{Protein kinase assay of PknF and PknG}

The ability of $\mathrm{PknF}$ and $\mathrm{PknG}$ to phosphorylate MBP was examined. Purified GST-PknF, GST-PknF-K41M, GST-PknG or GST-PknG-K181M were added to a reaction mixture containing $\mathrm{MBP}$ and $\left[\gamma_{-}{ }^{32} \mathrm{P}\right] \mathrm{ATP}$. After incubation, the products were separated by $15 \%$ SDSPAGE and the phosphorylated proteins were identified by autoradiography. Purified GST-PknF protein phosphorylated MBP, while mutant GST-PknF-K41M did not (Fig. 2a, lanes 3 and 2, respectively). Similarly, GST-PknG was able to phosphorylate MBP, while mutant GST-PknG-K181M had no effect (Fig. 2b, lanes 3 and 2, respectively). GST-PknF (Fig. 2a, lane 4) and GST-PknG (Fig. 2b, lane 4) were also autophosphorylated. GST-PknG exhibited less autophosphorylating ability than GST-PknF (Fig. 2b, lane 4 and Fig. 2a, lane 4, respectively). However, in the presence of MBP, autophosphorylation by $\mathrm{PknF}$ was markedly reduced whereas that of $\mathrm{PknG}$ was enhanced. It is well known that addition of proteins or peptides may enhance the activity of protein kinases (Hubler (a)

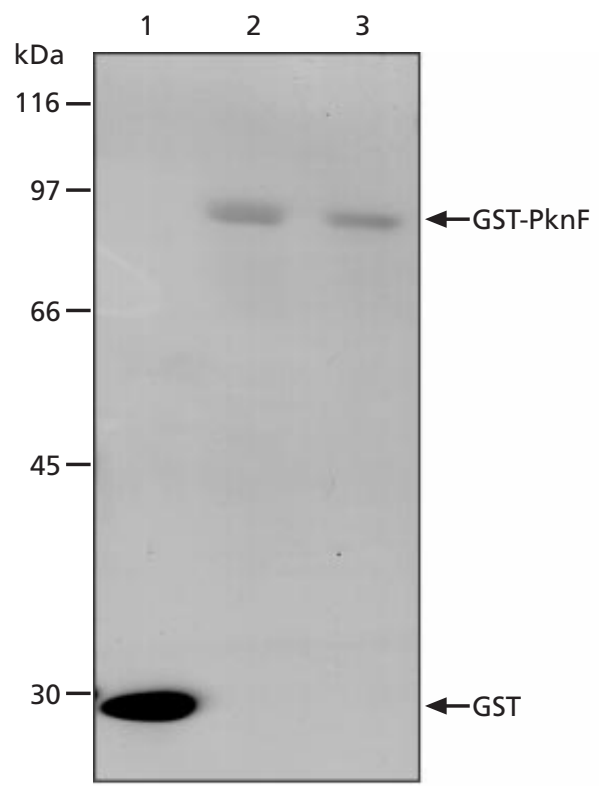

(b)

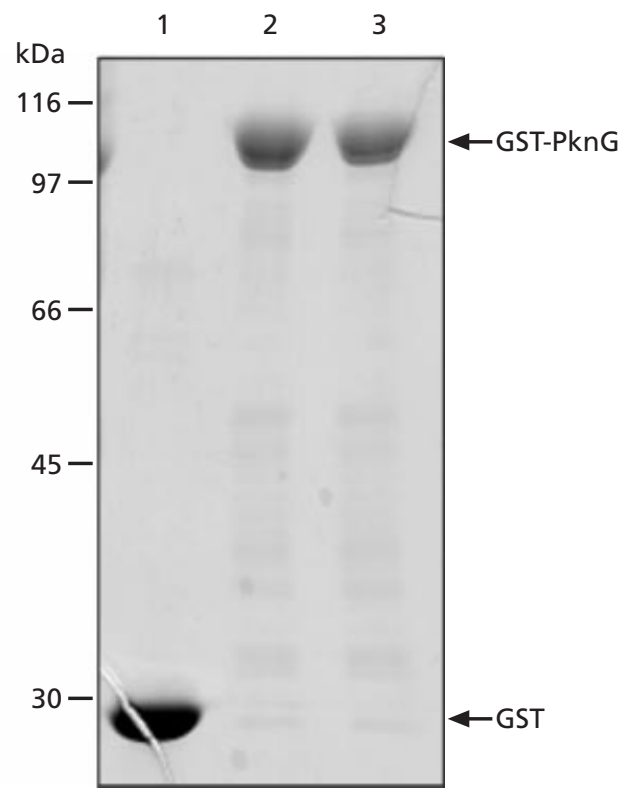

Fig. 1. Electrophoretic analysis of recombinant PknF (a) and PknG (b). Affinity-purified PknF and PknG were separated by $10 \%$ SDS-PAGE and stained with Coomassie blue. (a) Lanes: 1, GST alone; 2, GST-PknF chimeric protein; 3, GST-PknFK41M chimeric protein. (b) Lanes: 1, GST alone; 2, GST-PknG chimeric protein; 3, GST-PknG-K181M chimeric protein. Positions of molecular mass markers are indicated on the left. 
(a)

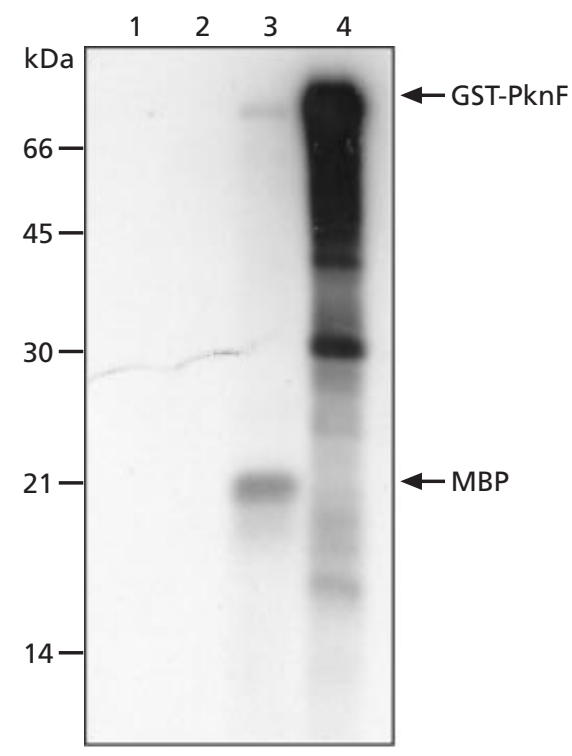

(b)

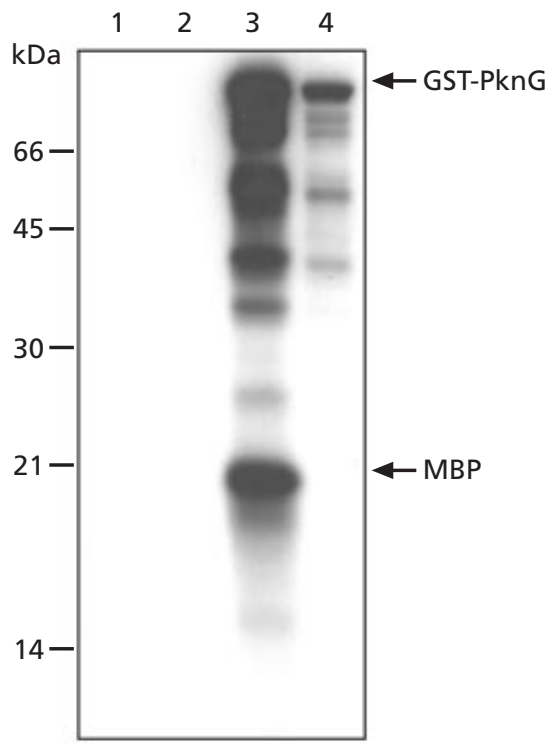

Fig. 2. Protein kinase activity of PknF (a) and PknG (b). Purified wild-type and mutant GST-PknF or GST-PknG (0.5 $\mu$ g) were incubated with MBP $(1 \mu \mathrm{g})$ in kinase buffer for $30 \mathrm{~min}$ at $37^{\circ} \mathrm{C}$. Proteins were separated by $15 \%$ SDS-PAGE, electroblotted and autoradiographed to determine phosphorylation of MBP. (a) Lanes: 1, MBP alone; 2, MBP plus mutant GST-PknF-K41M; 3, MBP plus wild-type GST-PknF; 4, GST-PknF alone. (b) Lanes: 1, MBP alone; 2, MBP plus mutant GST-PknG-K181M; 3, MBP plus wild-type GST-PknG; 4, GST-PknG alone. Positions of molecular mass markers are indicated on the left.

et al., 1992). To identify the residues of MBP phosphorylated by $\mathrm{PknF}$ or $\mathrm{PknG}$, phosphorylated MBP was acid-hydrolysed and analysed by twodimensional TLC. Incubation of MBP with GST-PknF phosphorylated serine and threonine residues of MBP (Fig. 3a), while GST-PknG phosphorylated MBP only at serine residues (Fig. $3 b$ ).

\section{Localization of PknF and PknG in mycobacterial cells}

Polyclonal antibodies raised against PknF and PknG were used to analyse the expression and localization of $\mathrm{PknF}$ in mycobacterial cells. Equal amounts of protein from cell wall, cell membrane and cytoplasmic subcellular fractions of M. tuberculosis $\mathrm{H}_{37} \mathrm{Rv}$, and wholecell lysates from M. smegmatis, M. tuberculosis $\mathrm{H}_{37} \mathrm{Rv}$ and M. tuberculosis $\mathrm{H}_{37} \mathrm{Ra}$ were separated by $10 \%$ SDSPAGE. The proteins were electroblotted onto a nitrocellulose membrane and incubated with anti-PknF serum or anti-PknG serum. The membranes were developed using an Enhanced Chemiluminescence kit. After development, $\mathrm{PknF}$ was seen as a doublet protein with an apparent molecular mass of $67-70 \mathrm{kDa}$ when recovered from cell wall (Fig. 4a, lane 1) or cell membrane (Fig. 4a, lane 2) fractions of M. tuberculosis $\mathrm{H}_{37} \mathrm{Rv}$. PknF was absent from cytoplasmic subcellular fractions (Fig. 4a, lane 3) of $M$ tuberculosis $\mathrm{H}_{37} \mathrm{Rv}$. However, the cytoplasmic fraction showed a band corresponding to $50 \mathrm{kDa}$, which may represent the unphosphorylated form of the protein. These results suggested that $\mathrm{PknF}$ is predominantly localized in the cell envelope of M. tuberculosis $\mathrm{H}_{37} \mathrm{Rv}$. PknF was also detected as a doublet protein of $67-70 \mathrm{kDa}$ in the wholecell lysates of M. tuberculosis $\mathrm{H}_{37} \mathrm{Rv}$ (Fig. 4a, lane 4) and M. tuberculosis $\mathrm{H}_{37} \mathrm{Ra}$ (Fig. 4a, lane 5), but not in those of M. smegmatis (Fig. 4a, lane 6).

The calculated molecular mass of $\mathrm{PknF}$ is $50.6 \mathrm{kDa}$, but during $10 \%$ SDS-PAGE it migrated as a doublet protein of $67-70 \mathrm{kDa}$. To check the specificity of anti-PknF serum, human lung epithelial cell line A549 was transfected with the pCDNA3-pknF construct, which encoded a translational product of $50 \cdot 6 \mathrm{kDa}$. Equal amounts of cell lysates from mock (pCDNA3 alone)and pCDNA3- $p k n F$-transfected A549 cell line were separated by $10 \%$ SDS-PAGE, transferred to a nitrocellulose membrane and incubated with anti-PknF serum. PknF was then seen as a double band of $67-70 \mathrm{kDa}$ after PAGE of protein extracts from pCDNA3-pknF transfected A549 cells (Fig. 4a, lane 8). This result was similar to that found with whole-cell lysates of $M$. tuberculosis $\mathrm{H}_{37} \mathrm{Rv}$ and $M$. tuberculosis $\mathrm{H}_{37} \mathrm{Ra}$ (Fig. 4a, lanes 4 and 5). The aberrant migration of PknF during SDS-PAGE may be due to post-translational modifications such as phosphorylation. Such an effect has been shown for many Ser/Thr protein kinases (Hanlon et al., 1997; Zhang, 1996; Motley \& Lory, 1999). A549 cells transfected with pCDNA3 vector alone (Fig. 4a, lane 7) failed to show bands that reacted with anti-PknF serum.

PknG was detected as an $82 \mathrm{kDa}$ protein predominantly in the cytoplasmic fraction of M. tuberculosis $\mathrm{H}_{37} \mathrm{Rv}$ 
(a)

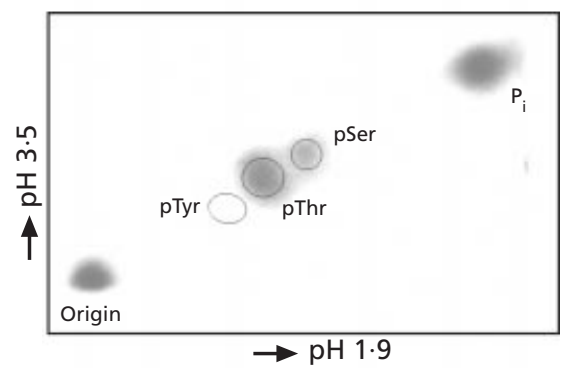

(b)

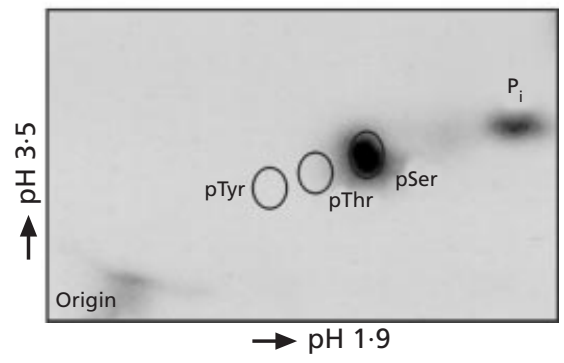

Fig. 3. Analysis of PknF-phosphorylated (a) and PknGphosphorylated (b) residues of MBP. Phosphorylated MBP residues were separated by $15 \%$ SDS-PAGE and electroblotted onto a PVDF membrane. Bands containing proteins were excised and hydrolysed in acid. The acid-stable phosphoamino acids liberated by hydrolysis were separated by twodimensional TLC and autoradiographed. ${ }^{32} \mathrm{P}_{i}$ was produced by partial hydrolysis of labelled amino acids. Samples of nonradioactive phosphotyrosine, phosphoserine and phosphothreonine were run in parallel and visualized by ninhydrin staining. PknF phosphorylates MBP on serine and threonine residues (a). PknG phosphorylates MBP on serine residues only (b).

(Fig. 4b, lane 3). PknG was also associated with cell membrane proteins (Fig. 4b, lane 2) of M. tuberculosis $\mathrm{H}_{37} \mathrm{Rv}$ and a faint band corresponding to $\mathrm{PknG}$ was detected in the cell wall fraction (Fig. $4 \mathrm{~b}$, lane 1). PknG expression could be detected in whole-cell lysates of $M$. tuberculosis $\mathrm{H}_{37} \mathrm{Rv}$ (Fig. 4b, lane 4) and $\mathrm{H}_{37} \mathrm{Ra}$ (Fig. 4b, lane 5), but not in whole-cell lysates from M. smegmatis (Fig. 4b, lane 6). Thus, the two proteins differ with respect to subcellular localization.

\section{Analysis of $p k n F$ and $p k n G$ in other mycobacterial strains}

The PCR products of $p k n F$ or $p k n G$ were used as probes in Southern hybridization experiments to determine the presence of $p k n F$ or $p k n G$ homologues among different species of mycobacteria. Genomic DNA from $M$. smegmatis, M. bovis BCG, M. tuberculosis $\mathrm{H}_{37} \mathrm{Rv}$ and M. tuberculosis $\mathrm{H}_{37} \mathrm{Ra}$ was digested with BamHI/NruI (for hybridization with $p k n F$ ) or with SmaI (for hybridization with $p k n G$ ). The digested fragments were separated by agarose gel electrophoresis and blotted onto nitrocellulose membranes. Hybridizations were (a)

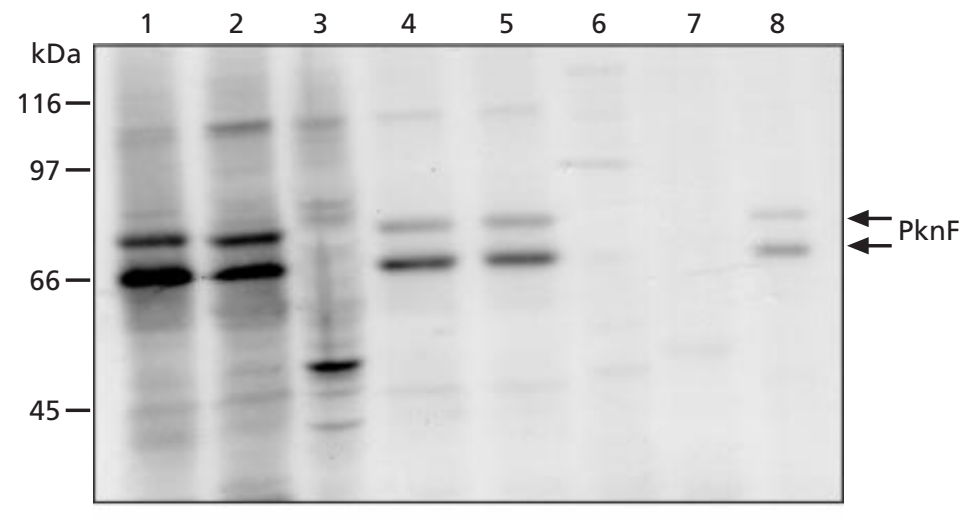

(b)

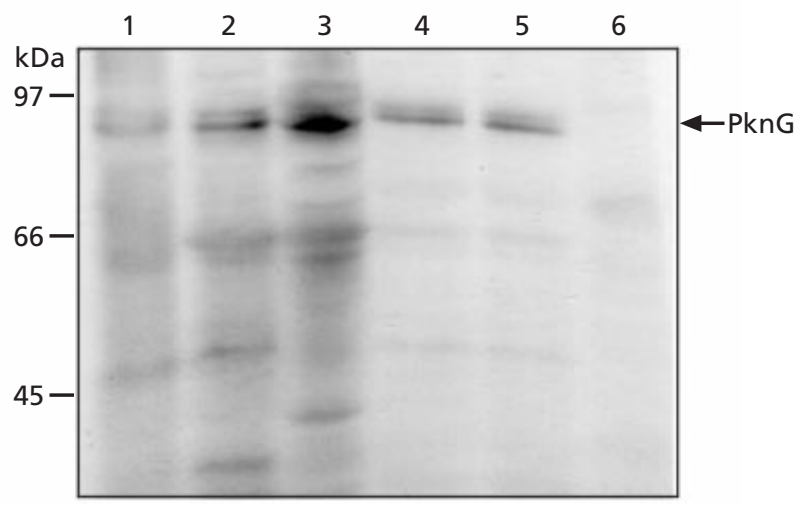

Fig. 4. Expression and localization of $P k n F(a)$ and $P k n G$ (b) in mycobacteria. Equal amounts of protein $(40 \mu \mathrm{g}$ each) of cell wall (lanes 1), cell membane (lanes 2) and cytoplasmic fraction (lanes 3) from $M$. tuberculosis $\mathrm{H}_{37} \mathrm{Rv}$, and whole-cell lysates from $M$. tuberculosis $\mathrm{H}_{37} \mathrm{Rv}$ (lanes 4), M. tuberculosis $\mathrm{H}_{37} \mathrm{Ra}$ (lanes 5) and $M$. smegmatis (lanes 6) were loaded onto a $10 \%$ SDS-polyacrylamide gel and electroblotted. The blots were probed either with anti-PknF antibodies (a) or anti-PknG antibodies (b) and developed using Enhanced Chemiluminescence regeants. Cell lysates from A549 cell lines transfected with pCDNA3 alone (a, lane 7) or with pCDNA3-pknF construct $(a$, lane 8$)$ were used to compare the molecular mass of the pknF gene translational product and PknF expressed protein in whole-cell lysates of $M$. tuberculosis $\mathrm{H}_{37} \mathrm{Rv}$ and M. tuberculosis $\mathrm{H}_{37} \mathrm{Ra}$. Positions of molecular mass markers are indicated on the left.

performed with a ${ }^{32} \mathrm{P}$-labelled $1350 \mathrm{bp} p k n F$ fragment or ${ }^{32}$ P-labelled 1747 bp $p k n G$ fragment, derived from PCR amplification and restriction digestion of the $p k n F$ $(B a m \mathrm{HI} / \mathrm{NruI})$ or $p k n G$ (SmaI) genes, respectively. Hybridization results revealed that genes homologous to $p k n F$ (Fig. 5a) and $p k n G$ (Fig. 5b) were present in members of the $M$. tuberculosis complex analysed in this study, but they were absent from M. smegmatis, a non-pathogenic mycobacterium. Interestingly, $p k n F$ hybridization also revealed one or two additional hybridization fragments of $2 \cdot 2 \mathrm{~kb}$ and $5.3 \mathrm{~kb}$ in the $M$. tuberculosis $\mathrm{H}_{37} \mathrm{Rv}$ and $\mathrm{H}_{37} \mathrm{Ra}$ lanes, respectively, consistent with the finding that PknF belongs to a family of 
(a)

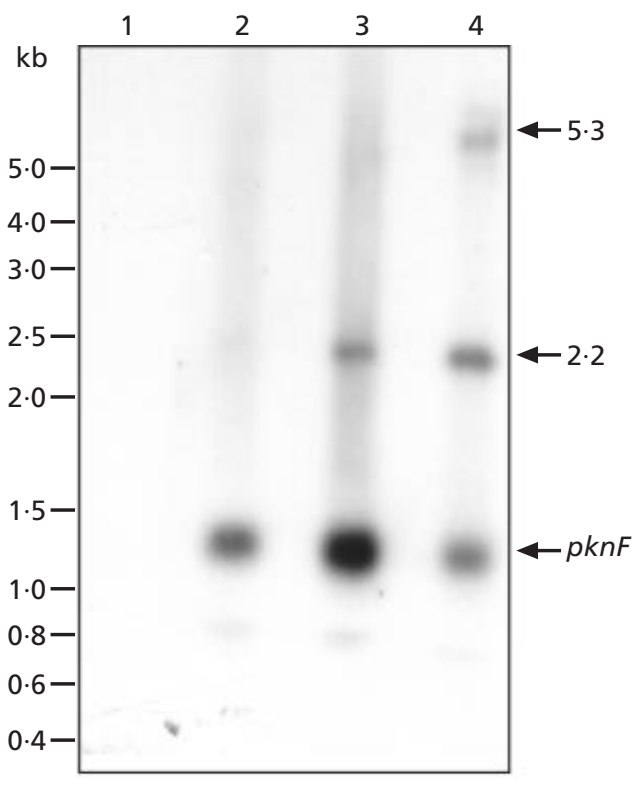

(b)

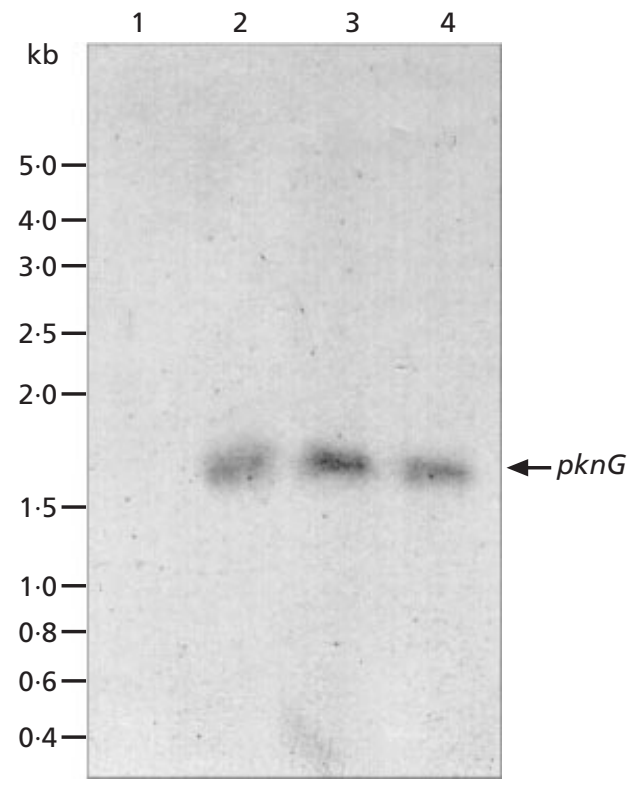

Fig. 5. Presence of $p k n F(a)$ and $p k n G$ (b) in other mycobacteria. Genomic DNA samples ( $3 \mu \mathrm{g}$ each) from mycobacterial species were digested with BamHI/Nrul (for hybridization with $p k n F$ ) or with Smal (for hybridization with $p k n G$ ) and the fragments were separated by $1 \%$ agarose gel electrophoresis for $16 \mathrm{~h}$ at $25-30 \mathrm{~V}$ and transferred to nitrocellulose membranes. Hybridization was performed with ${ }^{32}$ P-labelled $p k n F$ probe (a) or with ${ }^{32}$ P-labelled pknG probe (b) and autoradiographed. Lanes: 1, M. smegmatis; 2 , M. bovis BCG; 3, M. tuberculosis $\mathrm{H}_{37} R v$; 4, M. tuberculosis $\mathrm{H}_{37} R$. Positions of size markers are shown on the left.

related eukaryotic-like Ser/Thr protein kinases (Cole et al., 1998).

\section{DISCUSSION}

Phosphorylation of proteins at specific amino acid residues is a general mechanism by which both eukaryotes and prokaryotes regulate cell function. In prokaryotes, a two-component system consisting of a histidine kinase and its associated response regulator protein are used in sensing extracellular signals and coordinating intracellular events (Stock et al., 1989). In a number of prokaryotes Ser/Thr protein kinases also regulate development, response to stress conditions and pathogenicity (Av-Gay \& Davies, 1997). Examples include regulation of antibiotic production in Streptomyces coelicolor (Matsumoto et al., 1994), spore production in Myxococcus xanthus (Munoz-Dorado et al., 1991) and transduction of environmental signals in Bacillus subtilis (Yang et al., 1996). In these cases, the intracellular Ser/Thr kinases phosphorylate their target proteins within the bacterial cytoplasm. In contrast, an extracellular autophosphorylating protein kinase (YpkA) secreted by Yersinia species is translocated into the host cell cytoplasm where it phosphorylates host proteins and alters host cell function (Hakansson et al., 1996). Yersinia mutants deficient in YpkA are avirulent (Galyov et al., 1993).

Although phosphorylated proteins (Chow et al., 1994) and protein kinases (Av-Gay \& Everett, 2000; Peirs et al., 1997) have been found in mycobacteria, their role in mycobacterial pathogenesis is poorly understood. It has been well documented that $M$. tuberculosis has unique properties that allow it to remain in a dormant state within the host (Parrish et al., 1998). Protein kinases may play an important role in metabolic processes of $M$. tuberculosis. Therefore, we expressed and characterized two putative protein kinases, protein kinase $\mathrm{F}(\mathrm{PknF})$ and protein kinase $\mathrm{G}(\mathrm{PknG})$ of M. tuberculosis $\mathrm{H}_{37} \mathrm{Rv}$.

$\mathrm{PknF}$ and $\mathrm{PknG}$ have a characteristic protein kinase amino acid sequence signature, including all 11 conserved domains of Ser/Thr kinases (Hanks \& Quinn, 1991). The two enzymes phosphorylated MBP, an in vitro substrate for Ser/Thr kinases. All putative Ser/Thr kinases of mycobacteria, except for PknI, possess a lysine in the ATP-binding site (Av-Gay \& Everett, 2000). This feature is typical of Ser/Thr kinases (Av-Gay \& Everett, 2000). When lysine at position 41 (K41) of PknF or lysine at position 181 (K181) of PknG were substituted with methionine, the mutant proteins failed to phosphorylate MBP. These results suggest that $\mathrm{PknF}$ and $\mathrm{PknG}$ are functional kinases and that $\mathrm{K} 41$ of $\mathrm{PknF}$ or K181 of PknG are required for enzymic activity, consistent with their having the same catalytic properties as other Ser/Thr kinases.

Immunoblot analysis of various cellular fractions of $M$. tuberculosis $\mathrm{H}_{37} \mathrm{Rv}$ with anti-PknF serum revealed that $\mathrm{PknF}$ is localized mainly in the cell envelope. Also, predicted structural analysis of $\mathrm{PknF}$ from $M$. tuberculosis revealed that it has a transmembrane domain (Av- 
Gay \& Everett, 2000). These results suggest that PknF, like $\mathrm{PknB}$, is a transmembrane kinase and that it may serve as a receptor for environmental signals, as is known to be true for transmembrane kinases from Streptomyces and Myxobacteria (Nadvornik et al., 1999). It has also been postulated that since the $p k n F$ gene is located in the ATP-binding cassette transporter operon, it may be involved in phosphate transport (AvGay \& Everett, 2000).

The $p k n F$ ORF encoded a translational product of $50 \cdot 6 \mathrm{kDa}$, but Western blot analysis of whole-cell lysates of M. tuberculosis $\mathrm{H}_{37} \mathrm{Rv}$ and M. tuberculosis $\mathrm{H}_{37} \mathrm{Ra}$ revealed that PknF migrates during 10\% SDS-PAGE as a $67-70 \mathrm{kDa}$ doublet. Similar results were obtained with A549 cells transfected with pCDNA3-pknF. No deleterious effects were observed with transfected A549 cells. However, this does not rule out the possible involvement of $\mathrm{PknF}$ in virulence. The aberrant $\mathrm{mi}-$ gration of $\mathrm{PknF}$ during SDS-PAGE may be due to phosphorylation, as is observed in Ser/Thr kinase Pkn9 (Hanlon et al., 1997) and Pkn6 (Zhang, 1996) from Myxococcus xanthus and Ser/Thr kinase PpkA from Pseudomonas aeruginosa (Motley \& Lory, 1999). The migration of $\mathrm{PknF}$ as a doublet protein may reflect $\mathrm{PknF}$ molecules having different levels of phosphorylation.

In contrast to the transmembrane kinase $\mathrm{PknF}, \mathrm{PknG}$ is predominantly a cytoplasmic protein in M. tuberculosis $\mathrm{H}_{37} \mathrm{Rv}$. A cytoplasmic localization for PknG suggests that it is a soluble protein kinase. The secreted kinase (YopO) of Yersinia and PknG have similar structures (Av-Gay \& Everett, 2000), suggesting that PknG may have a role in the pathogenesis of mycobacteria. It has also been suggested from the genetic localization of $p k n G$ within the glutamate-binding operon that PknG may be involved in virulence by regulation of glutamate metabolism (Av-Gay \& Everett, 2000).

The genes encoding PknF and PknG, which were present in M. bovis BCG as well as in M. tuberculosis $\mathrm{H}_{37} \mathrm{Rv}$ and M. tuberculosis $\mathrm{H}_{37} \mathrm{Ra}$, were absent from the avirulent strain M. smegmatis. This observation is consistent with our Western blot data in which we failed to detect the expression of $\mathrm{PknG}$ or $\mathrm{PknF}$ in M. smegmatis culture lysates. The absence of $p k n F$ and $p k n G$ in nonpathogenic M. smegmatis suggests possible roles in the processes specific to pathogenic mycobacteria. More experimental evidence will be necessary to demonstrate a critical function of $\mathrm{PknF}$ or PknG in the pathology of tuberculosis, which could open new opportunities for the development of target-specific therapies.

\section{ACKNOWLEDGEMENTS}

We would like to express our gratitude to Dr John T. Belisle (Colorado, USA) for providing cell wall, cell membrane and cytosolic fraction proteins of $M$. tuberculosis $\mathrm{H}_{37} \mathrm{Rv}$ and whole-cell lysates of $M$. tuberculosis $\mathrm{H}_{37} \mathrm{Rv}$ and $M$. tuberculosis $\mathrm{H}_{37} \mathrm{Ra}$, and $\mathrm{Y}$. Dong for purification of $M$. tuberculosis DNA. Sincere thanks go to Norbert Prenzel, Johannes Bange, Reimar Abraham and Hemant Khanna for valuable discussions. A. K. was supported by the Council of
Scientific and Industrial Research (India) and DAAD (Germany).

\section{REFERENCES}

Armstrong, J. A. \& Hart, P. D. (1975). Phagosome-lysosome interactions in cultured macrophages infected with virulent tubercle bacilli. Reversal of the usual non-fusion pattern and observation on bactericidal survival. J Exp Med 142, 1-16.

Av-Gay, Y. \& Davies, J. (1997). Components of eukaryotic like signaling pathways in Mycobacterium tuberculosis. Microb Comp Genomics 2, 63-73.

Av-Gay, Y. \& Everett, M. (2000). The eukaryotic-like Ser/Thr protein kinases of Mycobacterium tuberculosis. Trends Microbiol 8, 238-244.

Av-Gay, Y., Jamil, S. \& Drews, S. J. (1999). Expression and characterization of the Mycobacterium tuberculosis serine/ threonine protein kinase PknB. Infect Immun 67, 5676-5682.

Chen, C. \& Okayama, H. (1987). High-efficiency transformation of mammalian cells by plasmid DNA. Mol Cell Biol 7, 2745-2752.

Chow, K., Ng, D., Stokes, R. \& Johnson, P. (1994). Protein tyrosine phosphorylation in Mycobacterium tuberculosis. FEMS Microbiol Lett 124, 203-208.

Cole, S. T., Brosch, R., Parkhill, J. \& 39 other authors (1998). Deciphering the biology of Mycobacterium tuberculosis from the complete genome sequence. Nature 393, 537-544.

Ferrari, G., Langen, H., Naito, M. \& Pieters, J. (1999). A coat protein on phagosomes involved in the intracellular survival of mycobacteria. Cell 97, 435-447.

Galyov, E. E., Hakansson, S., Forsberg, A. \& Wolf-Watz, H. (1993). A secreted protein kinase of Yersinia pseudotuberculosis is an indispensable virulence determinant. Nature 361, 730-732.

Hakansson, S., Gaylov, E. E., Rosqvist, R. \& Wolf-Watz, H. (1996). The Yersinia YpkA Ser/Thr kinase is translocated and subsequently targeted to the inner surface of the HeLa cell plasma membrane. Mol Microbiol 20, 593-603.

Hanks, S. \& Quinn, A. M. (1991). Use of degenerate oligonucleotide probes to identify clones that encode protein kinases. Methods Enzymol 200, 38-62.

Hanlon, W. A., Inouye, M. \& Inouye, S. (1997). Pkn9, a Ser/Thr protein kinase involved in development of Myxococcus xanthus. Mol Microbiol 23, 459-471.

Harlow, E. \& Lane, E. (1988). Antibodies: a Laboratory Manual, 1st edn. Cold Spring Harbor, NY: Cold Spring Harbor Laboratory.

Hubler, L., Leventhal, P. S. \& Bertics, P. J. (1992). Alteration of the kinetic properties of the epidermal growth factor receptor tyrosine kinase by basic proteins. Biochem J 281, 107-114.

Koul, A., Choidas, A., Treder, M., Tyagi, A. K., Drlica, K., Singh, Y. \& Ullrich, A. (2000). Cloning and characterization of secretory tyrosine phosphatases of Mycobacterium tuberculosis. J Bacteriol 182, 5425-5432.

Kunkel, T. A., Bebenek, K. \& McClary, J. (1991). Efficient sitedirected mutagenesis using uracil-containing DNA. Methods Enzymol 204, 125-139.

Laemmli, U. K. (1970). Cleavage of structural proteins during the assembly of the head of bacteriophage T4. Nature 227, 680-685.

Lowrie, D. B. (1983). How macrophages kill tubercle bacilli. J Med Microbiol 16, 1-12.

Matsumoto, A. S., Hong, S. K., Ishizuka, H., Horinouchi, S. \& Beppu, T. (1994). Phosphorylation of the AfsR protein involved in 
secondary metabolism in Streptomyces species by a eukaryotictype protein kinase. Gene 146, 47-56.

Motley, S. T. \& Lory, S. (1999). Functional characterization of a serine/threonine protein kinase of Pseudomonas aeruginosa. Infect Immun 67, 5386-5394.

Munoz-Dorado, J., Inouye, S. \& Inouye, M. (1991). A gene encoding a protein serine/threonine kinase is required for the normal development of $M$. xanthus, a gram-negative bacterium. Cell 67, 995-1006.

Nadvornik, R., Vomastek, T., Janecek, J., Technikova, Z. \& Branny, P. (1999). pKg2, a novel transmembrane protein Ser/Thr kinase of Streptomyces granaticolor. J Bacteriol 181, 15-23.

Parrish, N. M., Dick, J. D. \& Bishai, W. R. (1998). Mechanism of latency in Mycobacterium tuberculosis. Trends Microbiol 6, 107-112.

Peirs, P., Witt, L. D., Braibant, M., Huygen, K. \& Content, J. (1997). A serine/threonine protein kinase from Mycobacterium tuberculosis. Eur J Biochem 244, 604-612.

Prabhakaran, K., Harris, E. B. \& Randhawa, B. (2000). Regulation by protein kinase of phagocytosis of Mycobacterium leprae by macrophages. J Med Microbiol 49, 339-342.

Reyrat, J. M., Berthet, F. X. \& Gicouel, B. (1995). The urease locus of Mycobacterium tuberculosis and its utilization for the demonstration of allelic exchange in Mycobacterium bovis bacillus Calmette-Guerin. Proc Natl Acad Sci U S A 92, 8768-8772.

Sanger, F., Nicklen, S. \& Coulson, A. R. (1977). DNA sequencing with chain-terminating inhibitors. Proc Natl Acad Sci US A 74, 5463-5467.
Stock, J. B., Ninfa, A. J. \& Stock, A. M. (1989). Protein phosphorylation and regulation of adaptive responses in bacteria. Microbiol Rev 53, 450-490.

Sturgill-Koszycki, S., Schlesinger, P. H., Chakraborty, P. \& 7 other authors (1994). Lack of acidification in Mycobacterium phagosomes produced by exclusion of the vesicular proton-ATPase. Science 263, 678-681.

Vincent, C., Doublet, P., Grangeasse, C., Vaganay, E., Cozzone, A. J. \& Duclos, B. (1999). Cells of Escherichia coli contain a protein-tyrosine kinase, Wzc, and a phosphotyrosine-protein phosphatase, Wzb. J Bacteriol 181, 3472-3477.

Yang, X., Kang, C. M., Brody, M. S. \& Price, C. W. (1996). Opposing pairs of serine protein kinases and phosphatases transmit signals of environmental stress to activate a bacterial transcription factor. Genes Dev 10, 2265-2275.

Yarden, Y. \& Ullrich, A. (1988). Growth factor receptor tyrosine kinases. Annu Rev Biochem 57, 443-478.

Zhang, C. (1996). Bacterial signalling involving eukaryotic-type protein kinases. Mol Microbiol 20, 9-15.

Zwick, E., Wallasch, C., Daub, H. \& Ullrich, A. (1999). Distinct calcium-dependent pathways of epidermal growth factor receptor transactivation and PYK2 tyrosine phosphorylation in PC12 cells. J Biol Chem 274, 20989-20996.

Received 15 February 2001; revised 23 April 2001; accepted 25 April 2001. 